\title{
Age Dating of Globular Clusters Using UBV(RI) Main-sequence Photometry in the Two-color Diagram: Age of NGC 6397
}

\author{
Valery V. Kravtsov \\ Sternberg Astron. Ins., 13, University Ave., Moscow 119899, Russia
}

\begin{abstract}
I propose and apply a method for deriving ages of the metalpoor globular clusters (GCs) in a distance-independent way, which is based on an age indicator related to the main sequence in the two-color diagrams using the $U-B$ index. The age of the metal-poor GC NGC 6397 has been estimated, using Yale isochrones of Demarque et al. (1996), to be close to $16 \mathrm{Gyr}$ provided the cluster metallicity is near $[\mathrm{Fe} / \mathrm{H}]=-2.0$.
\end{abstract}

\section{Age Indicator, Isochrones and Observational Data}

The main sequence of metal-poor GCs exhibits a bend in the two-color dia$\operatorname{gram}(\mathrm{s})$. Analysis of the multicolor Yale isochrones of Demarque et al. (1996) shows that the color width of such a bend, i.e., a color difference between the turnoff point and main sequence at the same level of the $U-B$, is a function of age at a given metallicity. Hence the age of a GC can be derived by fitting isochrones of appropriate metallicity to the cluster two-color diagram(s). The best fit isochrone must show the most adequate reproduction of fiducial line of the sequence (mainly in its upper part) and location of its three basic points: points of the turnoff and main sequence at the same level of the $U-B$, and point of the extremum.

Multicolor photometry by Alcaino et al. (1997) of NGC 6397 has been used to apply this approach. The densest part of the cluster field and stars above the turnoff level were excluded from consideration. Since the most probable cluster metallicity (see Gratton et al. 2000; Castilho et al. 2000) is close to $[\mathrm{Fe} / \mathrm{H}]=-2.0$, the set of isochrones of appropriate metallicity was used.

\section{Result}

Panels of Fig. 1 show that the fitting criteria described above are best met by isochrone for $16 \mathrm{Gyr}$ age in each diagram presented. A fitting accuracy is of order $\pm 1 \mathrm{Gyr}$, taking into account the result that the best fit is approximately the same in all the diagrams. However, the real error in age may be as large as a few Gyr (VandenBerg 1999, private communication) even if the photometry is accurate, with no systematic effects. That may be due to uncertainty in the parameters of theoretical calculations such as $T_{\text {eff }}$, or to the problems of transformation of isochrones to the colors used, etc. (e.g., see, for details, Grundahl et al. 2000). Note that a change of $0.1 \mathrm{dex}$ in $[\mathrm{Fe} / \mathrm{H}]$ causes a change in the age estimate of 

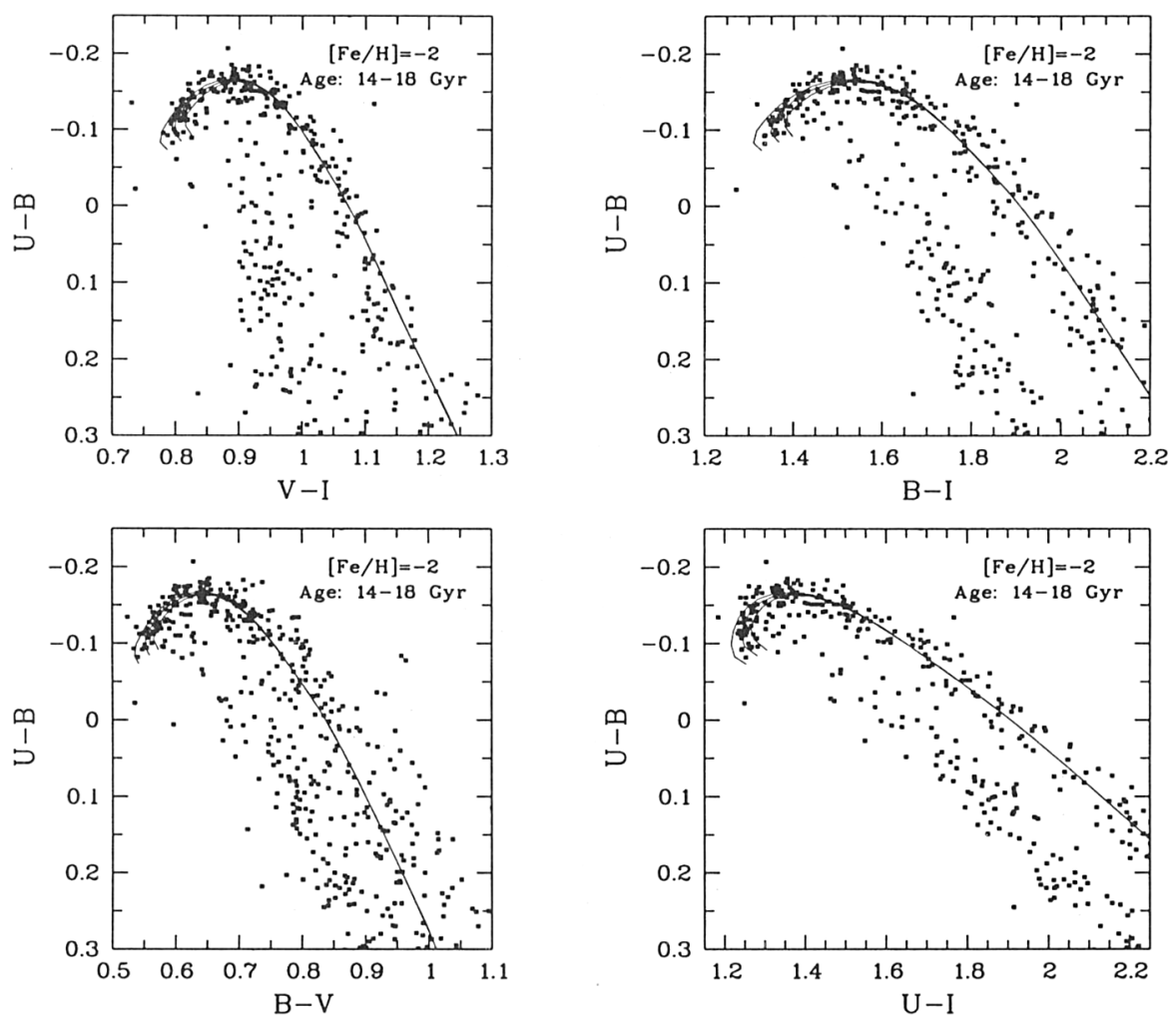

Figure 1. The two-color diagrams of NGC 6397, which show the main sequence with the superimposed Demarque et al. (1996) isochrones with an age of 14,16 , and $18 \mathrm{Gyr}, \mathrm{Y}=0.23,[\mathrm{Fe} / \mathrm{H}]=-2$. The panels demonstrate that the fitting criteria are best met in the diagrams by the isochrone for $16 \mathrm{Gyr}$ age. Two other isochrones are shown for comparison. Stars below the main sequence are field stars.

$\sim 1$ Gyr so that say for $[\mathrm{Fe} / \mathrm{H}]=-1.9$ the age derived for NGC 6397 is $\sim 15$ Gyr.

Acknowledgments. I am deeply thankful for the IAU travel grant. Also, I am very grateful to Dr. D. VandenBerg for valuable discussions and comments.

\section{References}

Alcaino, G., Liller, W., Alvarado, F., et al. 1997, AJ, 114, 1067

Castilho, B. V., Pasquini, L., Allen, D. M., et al. 2000, A\&A, 361, 92

Demarque, P., Chaboyer, B., Guenther, D., et al. 1996, Yale Isochrones

Gratton, R. G., Bonifacio, P., Bragaglia, A., et al. 2000, astro-ph/0012457

Grundahl, F., VandenBerg, D. A., Bell, R. A, et al. 2000, AJ, 120, 1884 